\title{
Transferencias tendíneas alrededor del hombro para paciente con secuelas de lesiones de plexo braquial
}

\author{
Tendon transfers around the shoulder for patient \\ with brachial plexus injury sequels \\ Guarachi JP, ${ }^{*, \neq}$ Bernal N, ${ }^{*, \neq}$ Calvo A,${ }^{\S}$ Paccot D, ${ }^{*, \neq, \S}$ Reinares $\mathrm{F}^{*, \neq, \S}$ \\ Clínica Alemana de Santiago, Santiago, Chile.
}

RESUMEN. Las lesiones de plexo braquial pueden afectar de forma muy significativa la función de los pacientes. El tipo de manejo va a depender de la severidad de la lesión como también de la localización anatómica de ésta. Las transferencias tendíneas alrededor del hombro han surgido como una alternativa efectiva de tratamiento y por lo tanto, debemos considerarlas cuando nos vemos enfrentados a pacientes que la podrían requerir. Existen diversas técnicas de transferencia, las que se utilizan con más frecuencia son las transferencias de trapecio superior, de dorsal ancho y de trapecio inferior, cada una con sus indicaciones y objetivos específicos según el tipo de paciente. Estas cirugías tienen como propósito disminuir el dolor producto de la hipotonía y subluxación glenohumeral como también mejorar el rango de movimiento del hombro, logrando que el paciente lleve su mano al plano que requiera para realizar sus actividades de la vida diaria. Presentaremos algunas de las técnicas de transferencia tendínea de hombro más utilizadas asociadas a una revisión bibliográfica y una descripción de nuestra experiencia con estas cirugías.

Palabras clave: Transferencias tendinosas, hombro, función, tratamiento, plexo braquial.

\section{Introducción}

Las lesiones de plexo braquial alteran de manera significativa la calidad de vida de los pacientes. Estas lesiones en los tiempos modernos son cada vez más frecuentes debido a accidentes de alta energía (automovilísticos, caídas de altura y deportes extremos).

\footnotetext{
* Departamento de Traumatología, Clínica Alemana de Santiago.

* Facultad de Medicina, Universidad del Desarrollo.

${ }^{\S}$ Departamento de Traumatología, Mutual de Seguridad.
}

Santiago, Chile.

https://dx.doi.org/10.35366/94626

doi: $10.35366 / 94626$
ABSTRACT. Brachial plexus lesions can significantly affect patient function. The type of management will depend on the severity of the injury as well as the anatomical location. Tendon transfers around the shoulder have emerged as an effective treatment alternative, and therefore we should consider them when faced with patients who might require it. There are various transfer techniques, within which the most frequently used are upper trapezium, latissimus dorsi and lower trapezium transfers, each with its specific indications and objectives depending on the type of patient. These surgeries aim to decrease the pain resulting from the hypotonia and glenohumeral subluxation as well as improve the range of movement of the shoulder, getting the patient to take his hand to the plane that requires to perform his daily life activities. We will provide a description of some of the most commonly used shoulder tendon transfer techniques associated with a bibliographic review and a description of our experience with these surgeries.

Keywords: Tendon transfers, shoulder, function, treatment, brachial plexus.

El manejo inicial de estos pacientes depende del tipo de lesión (neuropraxia, axonotmesis o neurotmesis) y la localización de la misma (preganglionar o postganglionar), el manejo médico a la espera de la recuperación espontánea es satisfactorio en la mayoría de los casos; sin embargo, algunos pacientes en quienes no hay una recuperación o el nivel de lesión impide que la recuperación neurológica sea

Dirección para correspondencia:

Felipe Reinares

Departamento de Traumatología, Clínica Alemana de Santiago.

Av. Manquehue Norte 1499, Piso 6,

Las Condes, Santiago, Chile.

E-mail: freinares@gmail.com

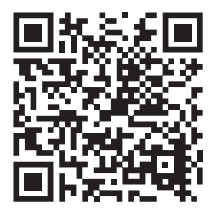


satisfactoria, es necesario utilizar técnicas microquirúrgicas como transferencias de nervios (neurotizaciones), trasplante de nervios o reconstrucción-reparación primaria. ${ }^{1,2,3}$

Una de las principales complicaciones o problemas secundarios de estas técnicas de reparación neurológica o del manejo médico de las mismas es el déficit de función completa o la pérdida de función parcial de los músculos y tendones alrededor del hombro, en especial del músculo deltoides, supraespinoso, infraespinoso y redondo menor, limitando elevación, abducción y especialmente la rotación externa (movimiento fundamental para tener un buen control de la mano en el espacio). ${ }^{4}$

Esto genera un patrón típico del paciente que se llama «mano sobre el abdomen» hand over belly (Figura 1). El paciente presenta una función parcial o completa de la mano y del codo, pero es incapaz de posicionar la mano en el plano coronal (debido al déficit de rotación y elevación del hombro). Esto limita de manera muy significativa el potencial de recuperación funcional, a pesar de la recuperación neurológica distal. ${ }^{4}$

Otro problema que genera importante dolor y disfunción de la extremidad, incluso siendo parte de la génesis del dolor neuropático persistente es la subluxación inferior de la articulación glenohumeral, la cual se establece por la pérdida del tono del músculo deltoides y del supraespinoso, activando receptores nociceptivos de la cápsula, lo que propicia la incidencia de dolor y rigidez secundaria.

Como alternativas quirúrgicas finales para el manejo del dolor en los pacientes con subluxación glenohumeral, pérdida de movilidad activa y pasiva, disquinesia escapular o escápula alada se plantean las opciones de la fusión o la artrodesis glenohumeral y la artrodesis escapulotorácica, éstas son completamente irreversibles y limitan de manera significativa la funcionalidad global de los pacientes. Se reservan para uso exclusivo de los pacientes con lesiones crónicas, inmanejables de manera médica y con contraindicación o falla de otros tratamientos.

Las transferencias tendinosas en pacientes con parálisis de hombro secundaria a lesiones del plexo braquial aparecen como alternativa intermedia (entre el manejo microquirúrgico y las fusiones o artrodesis), pero debido a la temporalidad hacen imposible o impredecible las alternativas de manejo quirúrgico del nervio o la obtención de resultados, a pesar de ello no son del todo satisfactorias o presentan déficit motor del hombro, especialmente la subluxación y el déficit de rotación externa. En este escenario se plantean como técnica complementaria o primaria las transferencias tendinosas alrededor del hombro, mediante las cuales se busca modificar quirúrgicamente la inserción y función de un músculo específico que no muestra compromiso neurológico, reemplazando la función de un músculo o grupo muscular comprometido con la lesión con el fin de recuperar de manera parcial o total la función perdida. ${ }^{5,6}$

Hay múltiples principios en la elección del músculo a transferir, pero lo más importante son sus características mecánicas, las cuales deben ser similares, éstas son: el vector de contracción, el potencial de excursión $(\mathrm{cm})$ y la tensión relativa (\%). Cuanto mayor sea su similitud, mejor será la función y más pronta la recuperación o reeducación motora de la misma. ${ }^{4}$

\section{Técnicas de transferencia muscular}

A continuación describiremos algunas de las técnicas más frecuentes encontradas en la literatura y las técnicas que utilizamos en nuestro medio para solucionar y poder ayudar a los pacientes con este diagnóstico:

1. Transfencia de trapecio superior: es posible que ésta sea la técnica históricamente más utilizada en pacientes con parálisis de plexo braquial adultos. Consiste en transferir o movilizar la porción superior del tendón distal del músculo trapecio del acromion lateral y espina escapular hacia el húmero proximal, distal a la tuberosidad mayor, reinsertándolo mediante una técnica de avance a través de una desinserción del músculo del deltoides (acromion lateral) con el fin de potenciar la función de elevación y abducción del hombro, reemplazando o potenciando la acción del deltoides. Esto aporta fuerzas de traslación superior de la cabeza humeral sobre la glenoides, favoreciendo el centraje glenohumeral, tratando la subluxación inferior.

Este procedimiento se puede realizar incluso después de una transferencia de ramas del nervio espinal accesorio a fibras del nervio supraescapular, neurotización muy frecuente para el manejo inicial de las lesiones de plexo braquial con compromiso de la función del hombro. Esta técnica fue descrita por Hoffa (1891), pero su principal modificación la hizo Saha (1967), de ahí que este procedimiento se conoce con este nombre ${ }^{7}$ (Figura 2). Rühmann (2008) describió

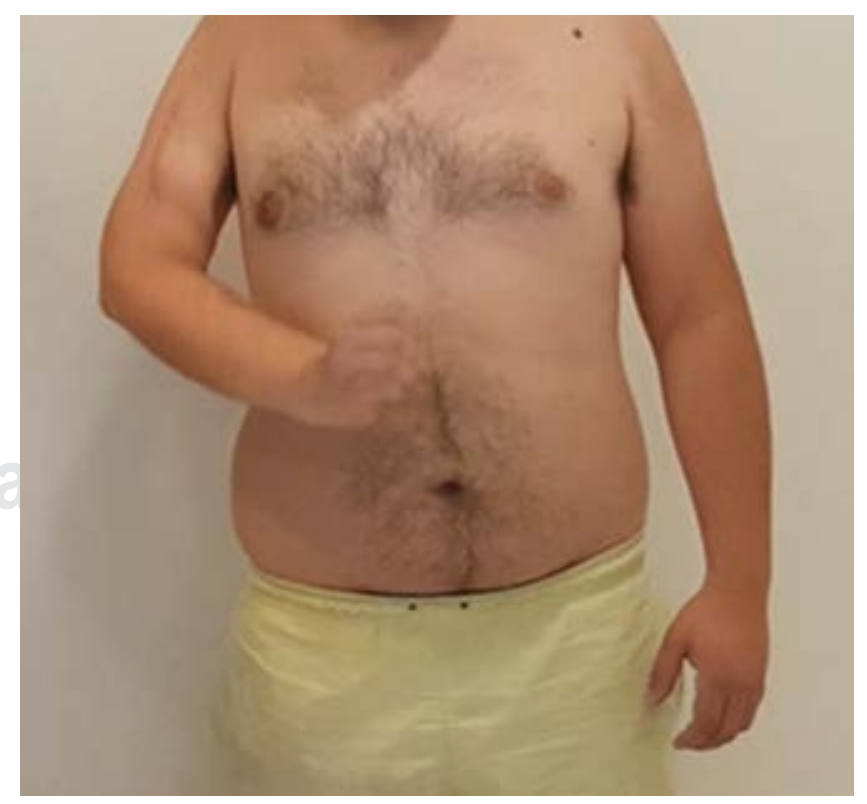

Figura 1: Postura clásica mano sobre el abdomen hand on belly de los pacientes con lesiones de plexo braquial. 
A

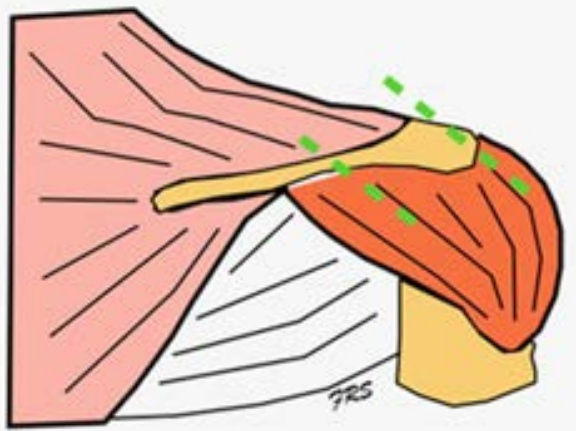

B

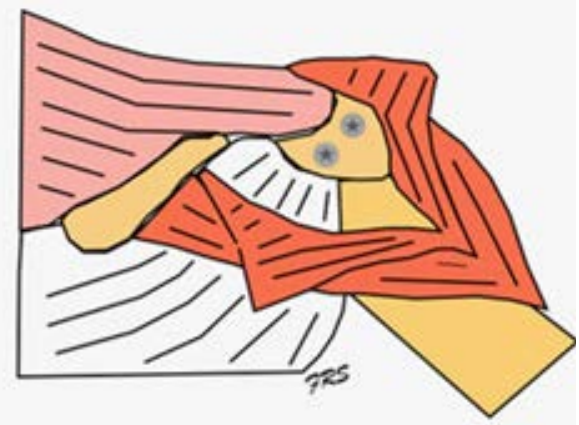

C

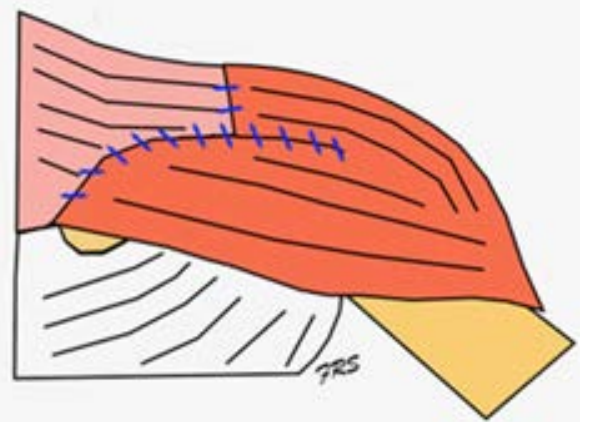

Figura 2: Técnica de Saha modificada. Transferencia de trapecio superior. A) Desinerción de deltoides y preparación de acromion lateral. B) Osteotomía y avance de trapecio superior más fijación de osteotomía mediante tornillos. C) Cierre de deltoides con técnica de avance V-Y.

la última modificación de la técnica con la que realiza una osteotomía del acromion lateral avanzando el tendón del trapecio superior (a través de un split del deltoides) y reinsertándolo mediante tornillos a la porción más distal de la tuberosidad mayor del húmero, posteriormente cierra el deltoides con una técnica V-Y, potenciando la contractibilidad remanente y tensión basal del mismo. Esta modificación favorece y aumenta las tasas de consolidación de la transferencia optimizando los resultados. Esta serie presenta mejoras significativas en cuanto a la subluxación inferior (de $95 \%$ a sólo $18 \%$ ). Sin embargo, los grados de ganancia de abducción y elevación anterior aumentan de manera discreta $\left(28^{\circ}\right.$ y $17^{\circ}$ respectivamente). ${ }^{8}$

Esta transferencia está orientada a disminuir la subluxación inferior y a mejorar parcialmente la abducción y elevación anterior, en ninguno de los reportes se describe de

manera precisa la recuperación de rotación externa del hombro. En nuestro medio no contamos con pacientes operados con esta técnica.

2. Transferencia de latisimus dorsi: la técnica de transferencia del latissimus dorsi o dorsal ancho asociado o no a la transferencia del redondo mayor fue descrita inicialmente en 1934 (l'Episcopo) para pacientes con parálisis obstétrica (parálisis de Erb) como consecuencia de tracción y lesión de las raíces $\mathrm{C} 5-\mathrm{C} 6-\mathrm{C} 7$ producida durante la salida del canal del parto. Se utiliza bajo condiciones muy específicas, donde hay déficit de rotación externa, contractura e hipertrofia del subescapular y cápsula anterior, pero no hay modificaciones óseas, secundaria al desbalance muscular y articular crónico, en un período crucial del desarrollo del niño.

Técnicamente lo que se realiza es un cambio de la inserción original del latissimus dorsi con o sin el redondo mayor (rotadores internos) ubicados en la cara anteromedial de la diáfisis humeral (medial a la corredera bicipital) hacia la cara lateral del húmero, pero rodeándolo por posterior insertando originalmente lateral a la corredera bicipital. Con esto se cambia el vector de función del músculo desde un rotador interno hacia un rotador externo ${ }^{9}$ (Figura 3).

A

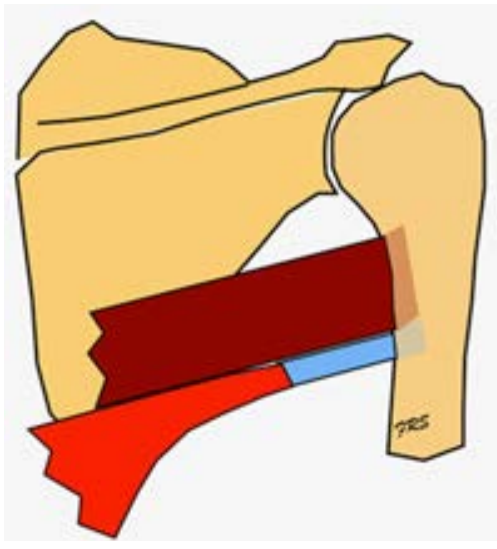

B

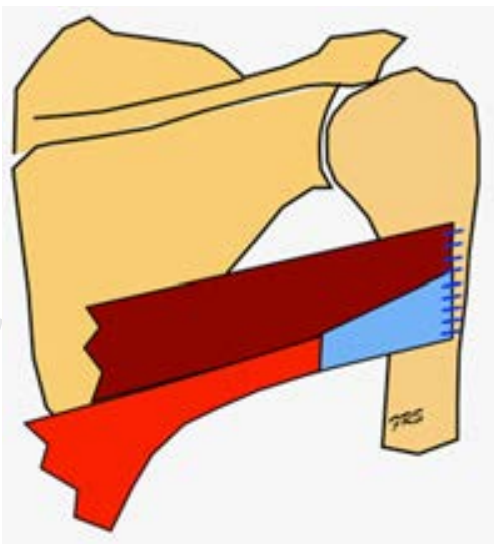

Figura 3: Esquema de técnica de l’Episcopo. A) Inserción anatómica del latissimus dorsi (LD) y redondo mayor (TM) en la región anteromedial del húmero diafisario. B) Representación postransferencia, cambio de inserción hacia cara posterolateral de húmero, rodeando la diáfisis por posterior. 

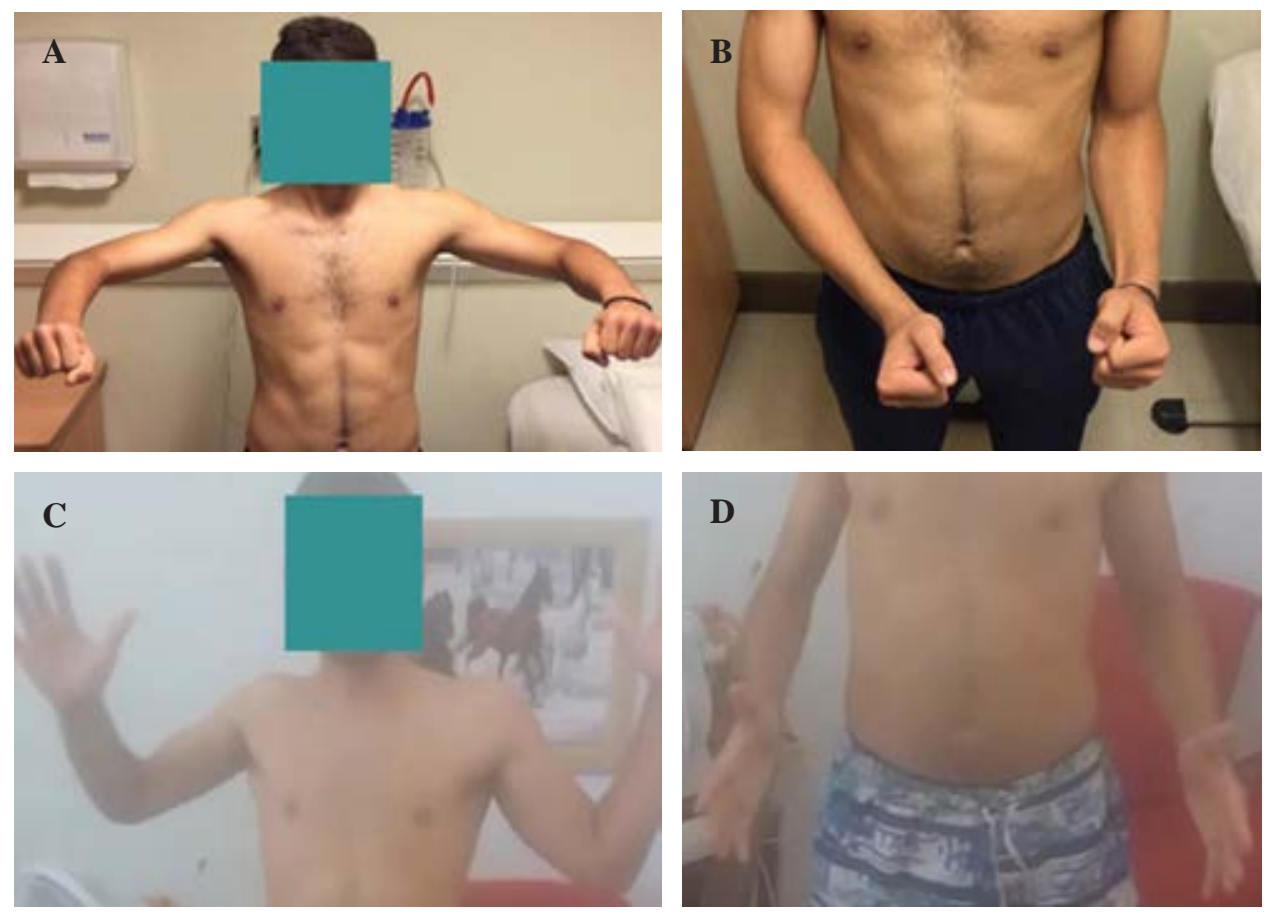

Figura 4:

Caso transferencia del latissimus dorsi modificada. A y B) Imágenes preoperatorias, rotación externa activa en posición abducta de $90^{\circ}$, donde obtiene $0^{\circ}$ y rotación externa activa en posición abducta, donde obtiene $-20^{\circ}$. C y D) Imágenes postoperatorias con dos años de seguimiento, rotación externa en posición de $90^{\circ}$, obtiene $70^{\circ} \mathrm{y}$ rotación externa activa en posición abducta obteniendo $10^{\circ}$.
Esta transferencia requiere un tendón del subescapular y un músculo deltoides competente porque de lo contrario estaría contraindicada, dado que es un importante depresor de la cabeza humeral (vector de tracción) con lo que podría exacerbar subluxaciones inferiores glenohumerales. Esta condición es poco frecuente en pacientes con lesiones traumáticas de plexo braquial. Sin embargo, es muy frecuente encontrar pacientes con parálisis obstétrica que cumplan con estos requerimientos.

En una serie reciente de Gosh (2013) con un gran número de pacientes adultos (24), la mayoría con lesiones de nivel C5-C6 de origen traumático, presentan resultados favorables en cuanto a la recuperación de rotación externa $\left(-28^{\circ}\right.$ a $\left.24^{\circ}\right)$, aumentando también la fuerza en rotación externa ( $0 \mathrm{lb}$ a 3.5 lb). Otro resultado importante es que en su serie 21/24 volvieron a trabajar y 13 al mismo trabajo prelesional. ${ }^{10}$

\section{Técnicas quirúrgicas}

A continuación, presentamos el caso de Sebastián (16 años), paciente brasileño que sufre de una parálisis obstétrica C5-C6 sin tratamiento previo, víctima de bullying producto de su posición anormal del brazo hand on belly. Clínicamente muestra una elevación anterior normal $\left(180^{\circ}\right)$, rotación externa de $-20^{\circ}$, más una disquinesia escapular severa. Después de rehabilitación kinésica de seis semanas realizó una técnica de transferencia del latissimus dorsi modificada, mediante una única incisión anterior de menos de $4 \mathrm{~cm}$ y una fijación lateral mediante túneles transóseos. A los dos años de seguimiento presenta una elevación anterior normal $\left(180^{\circ}\right)$ y una rotación externa de $+10^{\circ}$ grados (Delta $+30^{\circ}$ ) y una evaluación subjetiva del hombro muy satisfactoria (Figura 4).
Nuestra experiencia con la transferencia de este tendón ha sido muy satisfactoria; sin embargo, nuestra indicación principal es el déficit de elevación combinado con déficit de rotación externa parcial en los pacientes con patología degenerativa del manguito rotador. La idea de extrapolar la técnica original a los pacientes con lesiones degenerativas irreparables del manguito rotador fue descrita por Gerber (1981) y desde ese momento ha sufrido múltiples modificaciones, con buenos y excelentes resultados en cuanto a recuperación de la movilidad y disminución del dolor en series clínicas con más de 10 años de seguimiento. Actualmente utilizamos una técnica artroscópica y de fijación cortical tipo all inside similar a las técnicas de reconstrucción de ligamento cruzado anterior en la rodilla. Contamos con 17 pacientes operados con seguimiento mínimo de un año que presentan ganancia de elevación anterior de $54^{\circ}$, rotación externa de $11^{\circ}$, disminución de dolor de seis puntos y ganancia en escalas funcionales en promedio de 45 puntos (0-100).

3. Transferencia del trapecio inferior: probablemente es la transferencia que tiene resultados más promisorios en cuanto a la recuperación del control espacial de la extremidad, mejorando la función global. Descrita por Elhassan (2009) utiliza el músculo (trapecio inferior) que presenta mejor similitud mecánica con el principal rotador externo del hombro (infraespinoso), obteniendo un triple match (excursión, tensión relativa y vector muy similar), además de ser un músculo sinérgico en la acción de rotación externa nativa. Esto permite una alta potencialidad en cuanto a la recuperación de la función, con poco entrenamiento cerebral y reeducación motora. ${ }^{11}$ 
La técnica original se realiza mediante una doble incisión abierta (una medial a la escápula para la liberación y cosecha más una lateral para la reinserción tendínea). Debido a la diferencia entre la inserción original del trapecio y la inserción del infraespinoso es necesaria la expansión del tendón mediante un injerto alogénico (Aquiles) o autólogo (semitendinoso).

Elhassan (2014) presenta una serie de 111 pacientes tratados exclusivamente con transferencia de trapecio inferior o asociada a otras transferencias y ha obtenido $90 \%$ de resultados satisfactorios recuperando $70^{\circ}$ de rotación externa. A la luz de los buenos resultados obtenidos es probablemente una de las mejores alternativas para lograr recuperación aislada y rotación externa en este grupo de pacientes y así solucionar el problema de la «mano sobre el abdomen». ${ }^{12}$

En 2016 el mismo autor modifica su técnica realizando la cosecha del injerto mediante una incisión abierta medial al borde de la escápula, pero fijando el aloinjerto vía artroscópica, lo cual favorece la tasa de integración, reduce la lesión del músculo deltoides y disminuye las complicaciones postoperatorias relacionadas con el abordaje. ${ }^{13}$
Al igual que con la transferencia del latissimus dorsi, estos promisorios resultados llevaron a que la indicación se extrapolara al grupo de pacientes con roturas crónicas degenerativas e irreparables de los tendones del manguito rotador, cuyos resultados publicados son muy similares, logrando una rotación externa de $30^{\circ}$, ganancia de elevación de $50^{\circ}$ y disminución del dolor de manera significativa. ${ }^{13}$

En nuestro centro comenzamos la aplicación de esta técnica en los pacientes con roturas crónicas degenerativas del manguito rotador utilizando aloinjerto de Aquiles y una fijación artroscópica con botón cortical tipo All-inside (Figura 5). Contamos con una serie mixta (lesiones de plexo braquial y roturas tendíneas) de cinco pacientes con resultados muy similares a los descritos en la literatura disponible, con ganancia de rotación externa de $45^{\circ}$, con resultados buenos y excelentes en las evaluaciones subjetivas.

Hoy en día es nuestra herramienta principal para tratar pacientes adultos con lesiones traumáticas de plexo braquial, quienes claramente se beneficiarán de aumentar la rotación externa con el fin de tener un mejor control de la extremidad funcional remanente. Este grupo selecto de pacientes ha sido sometido previamente a múltiples cirugías:
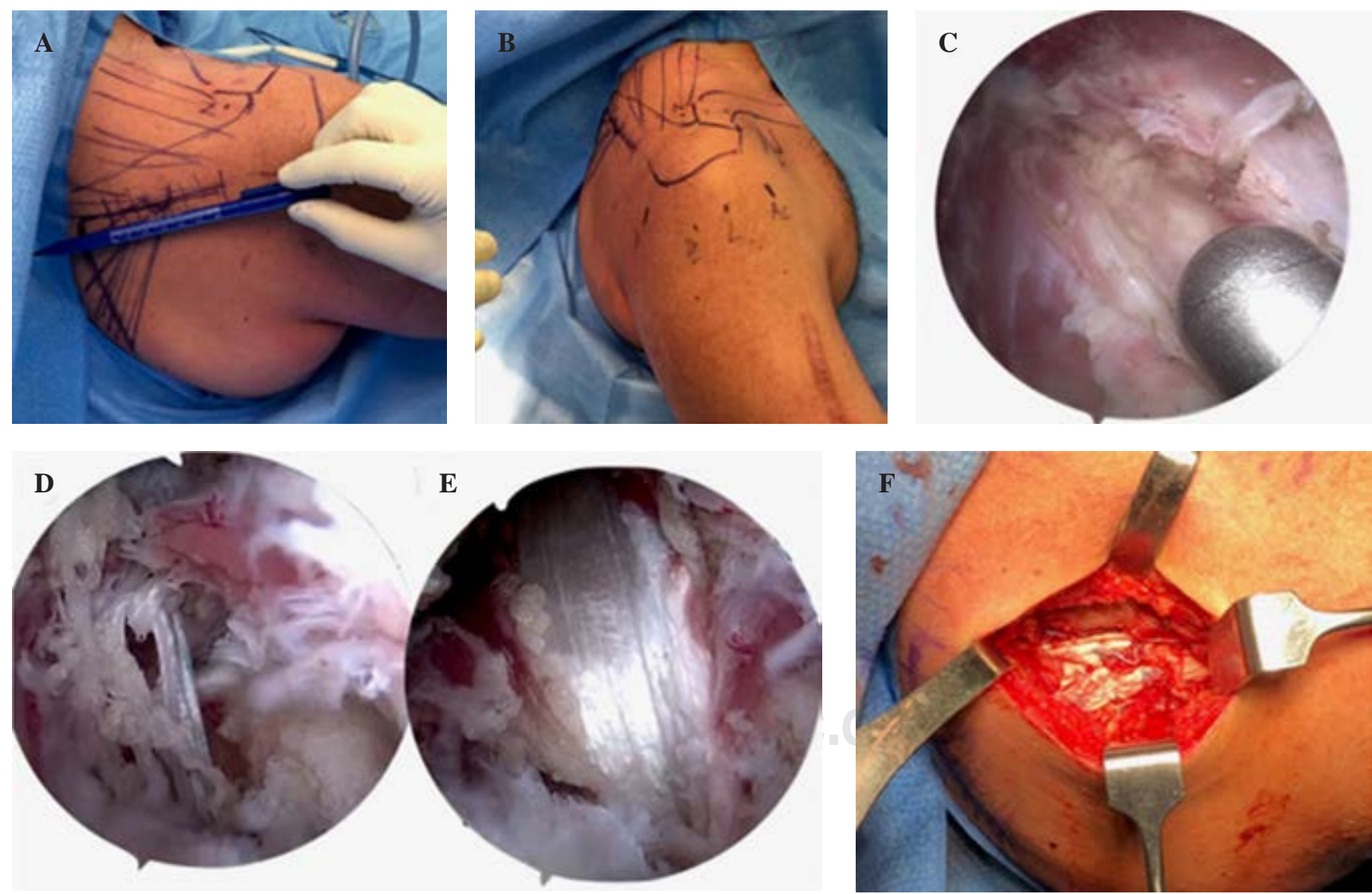

Figura 5: Técnica de trapecio inferior artroscópica aumentada con aloinjerto de Aquiles. A y B) Sitio de abordaje en espina escapular modificada (6 cm) y portales artroscópicos. C) Artroscopía, donde se observa el split del tendón del infraespinoso para realizar el túnel de inserción de la transferencia. D) Paso de suturas de fijación por túnel óseo para posterior tracción y fijación del aloinjerto de Aquiles. E) Visión de aloinjerto de Aquiles fijo en túnel a través del split del infraespinoso en posición muy satisfactoria. F) Vista final del aloinjerto de Aquiles fijo a tendón del trapecio inferior (mediante técnica tendón-tendón), con brazo rotado externo en 60 grados para obtener una tensión adecuada. 

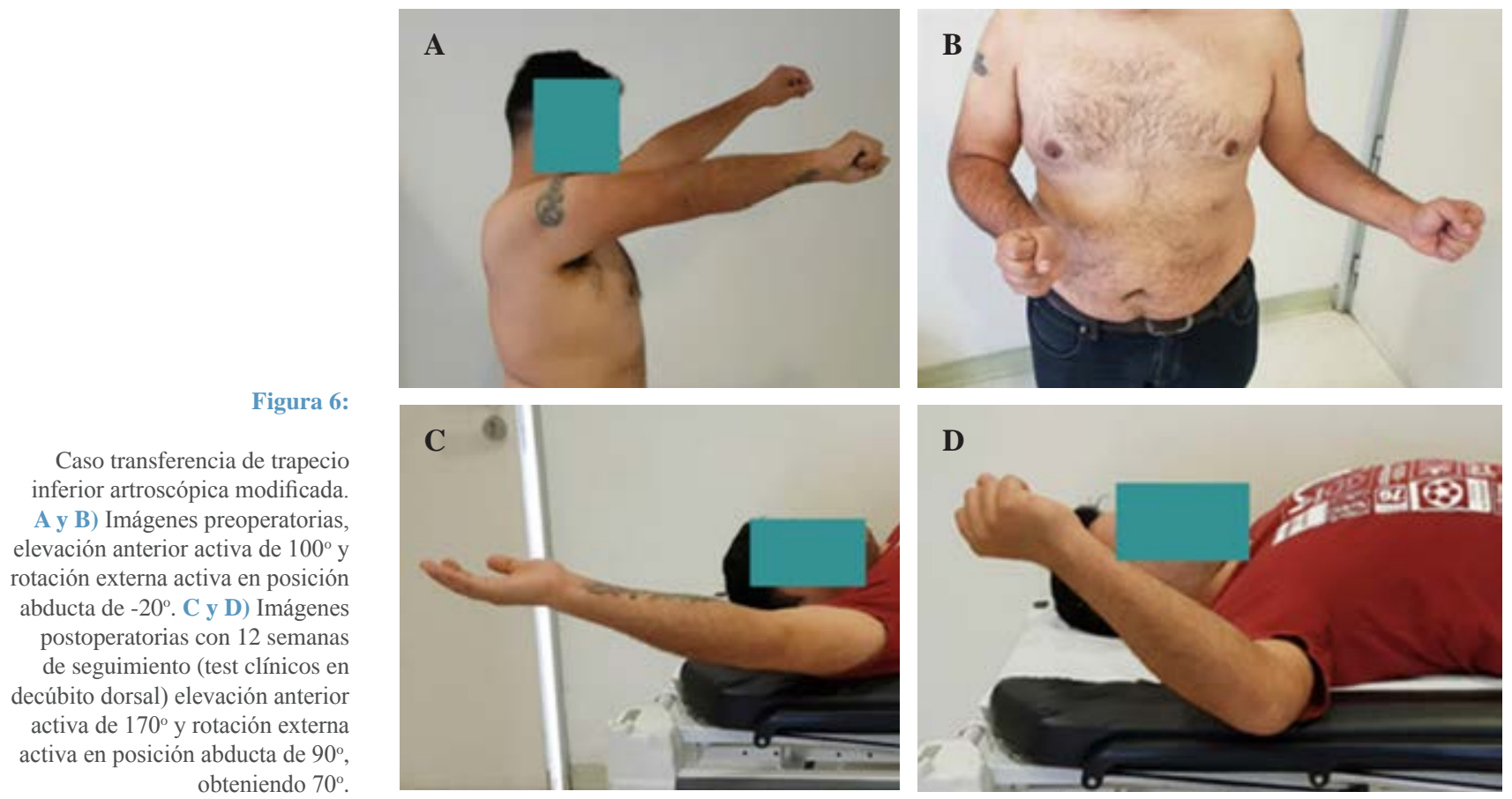

neurotizaciones, transferencias tendíneas de codo y mano. Esta técnica complementa el manejo y mejora la funcionalidad global de la extremidad.

A continuación presentamos el caso de Francisco (35 años) quien tuvo un accidente de alta energía (motocicleta). Como consecuencia padece de una lesión completa del tronco superior con un déficit parcial del nervio axilar y un déficit completo del nervio supraescapular. Función completa de codo y movilidad íntegra de la muñeca y mano. Fue tratado de manera conservadora, recuperando parcialmente algunos grados de elevación. Sin embargo, después de tres años de su lesión el paciente presenta una elevación anterior de $90^{\circ}$, una rotación externa de $-20^{\circ}$ y un síndrome Hand on belly. Se realizó una transferencia artroscópica del trapecio inferior, extendido con aloinjerto de Aquiles. Luego de tres meses de evolución (aún en etapa de rehabilitación) presenta una rotación externa de $30^{\circ}$ (en decúbito) y una elevación anterior de $150^{\circ}$ (decúbito), está muy satisfecho y contento con los resultados preliminares de su transferencia (Figura 6).

\section{Conclusión}

Actualmente las transferencias musculares en pacientes con lesiones de plexo braquial son una alternativa a nivel mundial en las que se preconiza el uso de una transferencia por objetivo específico. Transferencia del trapecio superior para recuperar elevación y subluxación inferior glenohumeral; transferencia del latissimus dorsi para el déficit de rotación externa en pacientes con parálisis obstétrica y transferencia de trapecio inferior para recuperar rotación externa de manera aislada.
Es importante considerar que este grupo de procedimientos se puede realizar de manera complementaria al tratamiento de las lesiones neurológicas específicas mediante cirugía de nervio o en el curso crónico de los pacientes cuando ya no son candidatos a éstas y uno quiere obtener o mejorar la funcionalidad lograda.

Hoy en día estas técnicas están disponibles para nuestros pacientes, los cuales tienen que ser muy bien seleccionados para obtener y poder replicar los excelentes resultados descritos en la literatura.

\section{Referencias}

1. Shin AY, Spinner RJ, Steinmann SP, Bishop AT. Adult traumatic brachial plexus injuries. J Am Acad Orthop Surg. 2005; 13: 38296.

2. Bertelli JA, Ghizoni MF. Transfer of the accessory nerve to the suprascapular nerve in brachial plexus reconstruction. J Hand Surg. 2007; 32A: 989-98.

3. Suzuki K, Doi K, Hattori Y, Pagsaligan JM. Long-term results of spinal accessory nerve transfer to the suprascapular nerve in uppertype paralysis of brachial plexus injury. J Reconstr Microsurg. 2007; 23: 295-9.

4. Elhassan B, Bishop A, Shin A, Spinner R. Shoulder tendon transfer options for adult patients with brachial plexus injury. J Hand Surg. 2010; 35A: 1211-19.

5. Narakas AO. Muscle transposition in the shoulder and upper arm for sequelae of brachial plexus palsy. Clin Neurol Neurosurg. 1993; 95(Suppl): 89-91.

6. Altmann S, Fansa H, Schneider W. Transfer of trapezius muscle for reconstruction of abduction of the shoulder. Orthopade. 2006; 35: 450-451, 453-5.

7. Saha AK. Surgery of the paralyzed and flail shoulder. Acta Orthop Scand. 1967; 97: 5-90.

8. Rühmann O, Kohn D, Bohnsack M. Modifizierte Technik der Verpflanzung des Musculus trapezius zur Funktionsverbesserung bei Armplexusschäden. Oper Orthop Traumatol. 2008; 20: 25-37. 
9. Waters PM. Update on the management of pediatric brachial plexus palsy. J Pediatr Orthop. 2005; 14B: 233-44.

10. Ghosh S, Singh VK, Jeyaseelan L, Sinisi M, Fox M. Isolated latissimus dorsi transfer to restore shoulder external rotation in adults with brachial plexus injury. Bone Joint J. 2013; 95-B: 660-3.

11. Elhassan B, Bishop AT, Shin A. Trapezius transfer to restore external rotation in a patient with brachial plexus injury: a case report. $J$ Bone Joint Surg. 2009; 91A: 939-44.

12. Elhassan B. Lower trapezius transfer for shoulder external rotation in patients with paralytic shoulder. J Hand Surg Am. 2014; 39 (3): 556-62.
13. Elhassan B, Alentorn-Geli E, Assenmacher AT, Wagner ER. Arthroscopic-assisted lower trapezius tendon transfer for massive irreparable posterior-superior rotator cuff tears: surgical technique. Arthrosc Tech. 2016; 5 (5): 981-8.

Conflicto de intereses: Los autores declaran que no existe conflicto de intereses. 